\title{
MYC Gene Rearrangements Are Closely Associated with Poor Survival of Diffuse Large B Cell Lymphoma with Hepatitis B Virus Infection
}

\author{
Zhihe Liu, ${ }^{1}$ Siyun Li, ${ }^{2}$ Wei Guo, ${ }^{1}$ Yinping Wang, ${ }^{3}$ Ming Wang, ${ }^{3}$ and Ou Bai ${ }^{1}$ \\ ${ }^{1}$ Department of Hematology, The First Hospital of Jilin University, Changchun 130021, China \\ ${ }^{2}$ Department of Pediatrics, Women and Children's Hospital of Qingdao University, Qingdao 266000, China \\ ${ }^{3}$ Department of Pathology Diagnosis Center, The First Hospital of Jilin University, Changchun 130021, China
}

Correspondence should be addressed to Ou Bai; oubai16@163.com

Received 2 August 2017; Revised 21 September 2017; Accepted 3 October 2017; Published 25 October 2017

Academic Editor: Carlo Visco

Copyright (C) 2017 Zhihe Liu et al. This is an open access article distributed under the Creative Commons Attribution License, which permits unrestricted use, distribution, and reproduction in any medium, provided the original work is properly cited.

\begin{abstract}
The aim of this study was to identify clinical adverse prognostic factors affecting overall survival (OS) of diffuse large B cell (DLBCL) patients with hepatitis B virus (HBV) infection. In this study, 30 DLBCL patients with HBV infection and 51 DLBCL patients with HBV-free were reviewed retrospectively. As of July 2016, the median follow-up period was 26.4 months (3.0 65.0 months). The median OS of patients in HBV infection group was 38.6 months, while that of patients in HBV-free group was not reached $(P=0.042)$; the median progression-free survival (PFS) of patients in HBV infection group was worse than that in HBV-free group, 18.5 months and 38.5 months $(P=0.118)$, respectively. The rate of $M Y C$ and $B C L 2$ gene rearrangements in $\mathrm{HBV}$ infection group was significantly higher than that in HBV-free group, $20.0 \%$ versus $3.9 \%(P=0.019)$ and $23.3 \%$ versus $5.9 \%(P=0.021)$, respectively. Multivariable analysis indicated that IPI $(P=0.002)$, chemotherapy regimens $(P=0.017)$, and $M Y C$ gene rearrangements $(P=$ 0.004 ) were independent adverse prognostic factors for all DLBCL patients in this study. Results demonstrated that the poor survival of DLBCL patients with HBV infection was closely involved in chemotherapy regimens, IPI, and MYC gene rearrangements.
\end{abstract}

\section{Introduction}

HBV infection remains a serious public health problem. It is reported that there are nearly 2 billion people currently suffering from HBV infection, and some 360 million are believed to be infected with chronic HBV infection worldwide, which includes 93 million people in China [1-3]. Therefore, China currently is still a region where $\mathrm{HBV}$ is endemic. Furthermore, there are as high as 300,000 deaths per year caused by HBV-related diseases [4].

To the best of our knowledge, the pathogenesis of many diseases is closely associated with HBV infection. At present, it has already been confirmed that HBV infection can remarkably increase the incidence of lymphomas, especially DLBCL [5-7]. What is more, some researchers discover that the survival of DLBCL patients with $\mathrm{HBV}$ infection is poor, compared to those DLBCL patients without HBV infection $[8,9]$. But to date, there are few articles systematically investigating clinical adverse prognostic factors of DLBCL patients with HBV infection. Therefore, the purpose of this study is to explore clinical adverse prognostic factors of DLBCL patients with HBV infection.

\section{Materials and Methods}

2.1. Patient Selection. A total of 201 patients initially diagnosed with DLBCL-NOS between January 1, 2011, and December 31, 2015, in the First Hospital of Jilin University were retrospectively reviewed. Of 201 patients, 182 patients had complete clinical information and received the first-line chemotherapy, such as R-CHOP, CHOP, and CHOP-like regimens. Patients with DLBCL transforming from low-grade lymphomas and those with primary cutaneous and primary central nervous system DLBCL, Epstein-Barr virus positive $\left(\mathrm{EBV}^{+}\right) \mathrm{DLBCL}$, and hepatitis $\mathrm{c}$ virus infection $\left(\mathrm{HCV}^{+}\right)$ 
DLBCL were excluded, and 81 patients were recruited in this study finally.

We retrospectively reviewed the results of HBV infection of all patients in this present study from our department of laboratory. Based on the results of HBV serum test, 30 patients were assigned to HBV infection group as the hepatitis B surface antigen was positive; 51 patients were assigned to HBV-free group (1, all HBV serum markers were negative; 2, HBV surface antibody positive and other serum markers negative should also be considered as HBV-free since some patients might receive HBV vaccine). All patients' diagnoses were in line with the lymphatic hematopoietic system malignant tumor classification standard (WHO, 2008) [10], and all patients' clinical stages were in accordance with the Ann Arbor staging system [11]. The study was conducted in accordance with the Helsinki Declaration, and the protocol had been approved by the ethics review committee of the First Hospital of Jilin University. Written informed consent was obtained from the patients or their legal guardians before carrying out the study.

2.2. Prognostic Factors. Prognostic factors included age, sex, clinical stage, molecular subtype (GCB and non-GCB), IPI, Ki-67, MYC gene rearrangements, BCL2 gene rearrangements, and chemotherapy regimens. The GCB, non-GCB, and $\mathrm{Ki}-67$ were examined by immunohistochemistry, which were routinely detected in Pathology Diagnosis Center. The other information, including age, sex, clinical stage, IPI, and chemotherapy regimens, was retrospectively collected in the database of the First Hospital of Jilin University. The immunohistochemistry results were evaluated blindly by two hematopathologists based on the current World Health Organization criteria and Hans algorithm, respectively [10, 12].

2.3. I-FISH for MYC and BCL2 Gene. The gene rearrangements of MYC and BCL2 were detected by interphase fluorescence in situ hybridization (I-FISH) on formalin-fixed and paraffin-embedded (FFPE) lymphoma samples of 81 DLBCL patients using $M Y C$ and BCL2 gene dual-color, break-apart probes (Vysis, Abbott Molecular, USA); more than 100 nuclei were examined for each probe whenever possible. 20 cases of patients with necrotic lymph node inflammation were selected as well to estimate the cutoff value of MYC and $B C L 2$ gene rearrangements. The cutoff value for the dualcolor break-apart arrangement probes was established by evaluating the split signal distribution in samples of reactive lymphoid tissues, calculating the mean number of split signals plus 3 times the standard deviation; the cutoffs value for $M Y C$ and BCL2 gene rearrangements was $8.9 \%$ and $9.8 \%$, respectively.

2.4. Treatments. Before receiving chemotherapy, real-time quantitative polymerase chain reaction (RT-PCR) was used to test the HBV-DNA copy number. For patients with HBVDNA copy number more than $1000 \mathrm{IU} / \mathrm{ml}$, they did not receive chemotherapy until their $\mathrm{HBV}$-DNA copy number was less than $1000 \mathrm{IU} / \mathrm{ml}$ after antivirus therapy (Lamivudine or Entecavir). In this study, all patients in $\mathrm{HBV}$ infection group received antivirus prophylaxis from initial chemotherapy to at least three months after completion of last chemotherapy for preventing HBV reactivation.

All patients achieved 2-8 cycles of first-line treatment, such as R-CHOP, CHOP, and R-CHOP/CHOP-like chemotherapy; for patients with progressive disease after firstline chemotherapy, they received second-line chemotherapy, for example, ICE, Gmox, GDP, ESHAP, DICE, and DHAP regimens. In order to monitor $\mathrm{HBV}$ reactivation, $\mathrm{HBV}$-DNA copy number of patients in HBV infection group was performed before each course of chemotherapy.

2.5. Survival. OS was defined from diagnosis to death or last follow-up. PFS was defined from diagnosis to disease progression, death, or last follow-up.

2.6. Statistics. SPSS 20.0 software was used for the statistical analysis, and OS was analyzed using the Kaplan-Meier method. Single variable and multivariable analyses were performed by Cox regression analysis. General information and clinical efficacy were compared by the $X^{2}$ test. $P$ value $\leq 0.05$ was regarded as statistical significance.

\section{Results}

3.1. Base Information. In this cohort, the prevalence of $\mathrm{HBV}$ infection among these DLBCL patients was $16.5 \%$ (30/182). In $\mathrm{HBV}$ infection group, the median duration time for antivirus prophylaxis was 8 months (5-13 months); out of these 30 DLBCL patients with $\mathrm{HBV}$ infection, $70.0 \%$ patients had Lamivudine therapy from initial chemotherapy to at least three months after completion of last chemotherapy, and 30.0\% received Entecavir therapy; unfortunately, there were still three patients suffering from $\mathrm{HBV}$ reactivation during chemotherapy. Of these three patients, two received rituximab-containing chemotherapy and one adopted chemotherapy without rituximab, the rate of $\mathrm{HBV}$ reactivation was $10.0 \%(3 / 30)$. The first patient had $\mathrm{HBV}$ reactivation after the fifth cycle of rituximab-containing chemotherapy combined with Lamivudine therapy; the second patient contracted HBV reactivation after the third cycle of chemotherapy without rituximab combined with Lamivudine therapy; the last patient suffered from $\mathrm{HBV}$ reactivation after the second cycle of rituximab-containing chemotherapy combined with Lamivudine therapy. These three patients discontinued chemotherapy after HBV reactivation and continued antivirus therapy; it is gratifying that the efficacy of antivirus was good and they successfully completed chemotherapy; up to now, they were in good condition. There was no statistical significance for age, sex, stage, IPI, Ki67 , molecular subtype, and chemotherapy regimens between $\mathrm{HBV}$ infection group and HBV-free group (Table 1).

3.2. MYC and BCL2 Gene Rearrangements. MYC and BCL2 gene rearrangements of all patients were analyzed by I-FISH in this study. In HBV infection group, the rate of MYC gene rearrangements was $20.0 \%$, which was higher than that (3.9\%) in HBV-free group and there was a statistical significance between the two groups $(P=0.019)$ (Figures 1 and 2 ). 
TABLE 1: Patient and disease characteristics.

\begin{tabular}{|c|c|c|c|}
\hline & HBV infection group $(n=30)$ & HBV-free group $(n=51)$ & $P$ value \\
\hline \multicolumn{4}{|l|}{ Age } \\
\hline$\geq 60$ & $9(30.0 \%)$ & $20(39.2 \%)$ & \multirow{2}{*}{0.403} \\
\hline$<60$ & $21(70.0 \%)$ & $31(60.8 \%)$ & \\
\hline \multicolumn{4}{|l|}{ Sex } \\
\hline male & $18(60.0 \%)$ & 27 (52.9\%) & \multirow{2}{*}{0.537} \\
\hline female & $12(40.0 \%)$ & $24(47.1 \%)$ & \\
\hline \multicolumn{4}{|l|}{ Stage } \\
\hline I/II & $12(40.0 \%)$ & $23(45.1 \%)$ & \multirow{2}{*}{0.655} \\
\hline III/IV & $18(60.0 \%)$ & $28(54.9 \%)$ & \\
\hline \multicolumn{4}{|l|}{ IPI } \\
\hline$>2$ & $11(36.7 \%)$ & $21(41.2 \%)$ & \multirow{2}{*}{0.688} \\
\hline$\leq 2$ & $19(63.3 \%)$ & $30(58.8 \%)$ & \\
\hline \multicolumn{4}{|l|}{ Ki-67 } \\
\hline$>80 \%$ & $10(33.3 \%)$ & $9(17.6 \%)$ & \multirow{2}{*}{0.108} \\
\hline$\leq 80 \%$ & $20(66.7 \%)$ & $42(82.4 \%)$ & \\
\hline \multicolumn{4}{|c|}{ Molecular subtype } \\
\hline GCB & $8(26.7 \%)$ & $14(27.5 \%)$ & \multirow{2}{*}{0.939} \\
\hline Non-GCB & $22(73.3 \%)$ & $37(72.5 \%)$ & \\
\hline \multicolumn{4}{|l|}{ Rituximab } \\
\hline Yes & $13(43.3 \%)$ & $33(64.7 \%)$ & \multirow{2}{*}{0.061} \\
\hline No & $17(56.7 \%)$ & $18(35.3 \%)$ & \\
\hline \multicolumn{4}{|c|}{ Antivirus therapy } \\
\hline Lamivudine & $21(70.0 \%)$ & 0 & \multirow{2}{*}{ NA } \\
\hline Entecavir & $9(30.0 \%)$ & 0 & \\
\hline
\end{tabular}

$\mathrm{NA}=$ no application.

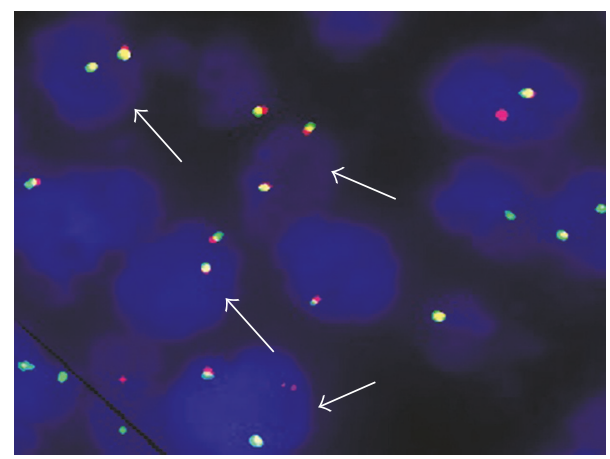

Figure 1: DLBCL patients with normal gene (arrow indicated lymphoma cells without gene rearrangements).

The rate of BCL2 gene rearrangements in HBV infection group was nearly 4 -fold higher than that in $\mathrm{HBV}$-free group (23.3\% versus $5.9 \%$ ), and there was also a statistical significance between the two groups $(P=0.021)$ (Table 2$)$. In this study, there was one "double-hit" patient harboring MYC and $B C L 2$ gene rearrangements in $\mathrm{HBV}$ infection group.

3.3. Survival. As of July 2016, the median follow-up period was 26.4 months (3.0 65.0 months). At the end of followup, the median OS of patients in HBV infection group was 38.6 months, while the median OS of patients in HBV-free

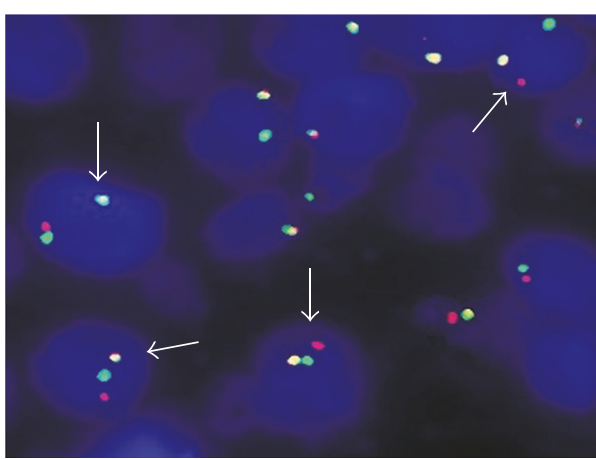

FIGURE 2: DLBCL patients with gene rearrangements (arrow showed lymphoma cells with gene rearrangements).

group was not reached. There was significant difference for OS between the two groups $(P=0.042)$ (Figure 3$)$. Similarly, the median PFS of patients in HBV infection group was worse than that in HBV-free group, 18.5 months and 38.5 months $(P=0.118)$, respectively (Figure 4$)$.

We further analyzed OS and PFS of patients between rituximab-containing chemotherapy and rituximab-free chemotherapy, and results indicated that the median OS of patients in HBV infection group was worse than that in HBV-free group, 41.6 months versus not reached $(P=0.032)$, respectively (Figure 5); similarly, the median PFS of patients 
TABLE 2: The rate of MYC and BCL2 gene rearrangements.

\begin{tabular}{|c|c|c|c|}
\hline Gene rearrangements & HBV infection group $(n=30)$ & HBV-free group $(n=51)$ & $P$ value \\
\hline \multicolumn{4}{|l|}{$M Y C$} \\
\hline Positive & $6(20.0 \%)$ & $2(3.9 \%)$ & \multirow{2}{*}{0.019} \\
\hline Negative & $24(80.0 \%)$ & $49(96.1 \%)$ & \\
\hline \multicolumn{4}{|l|}{$B C L 2$} \\
\hline Positive & $7(23.3 \%)$ & $3(5.9 \%)$ & \multirow{2}{*}{0.021} \\
\hline Negative & $23(76.7 \%)$ & $48(94.1 \%)$ & \\
\hline
\end{tabular}

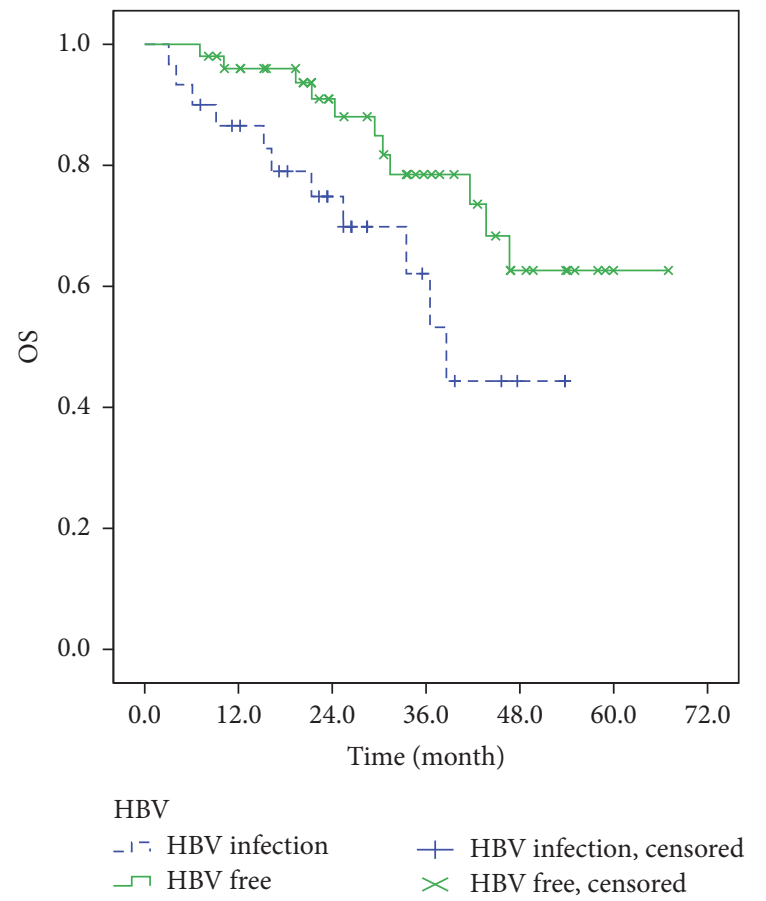

FIGURE 3: The overall survival of DLBCL patients in HBV infection group and HBV-free group.

in HBV infection group was also worse than that in HBVfree group, 20.2 months versus not reached $(P=0.042)$, respectively (Figure 6).

3.4. Analysis of Independent Risk Factors. Single variable, including sex, age, clinical stage, IPI, Ki-67, molecular subtype, chemotherapy regimens, MYC and BCL2 gene rearrangements, and HBV status were analyzed by Cox regression analysis in this study, and results revealed that IPI $(P=$ $0.003)$, chemotherapy regimens $(P=0.038)$, MYC gene rearrangements $(P=0.005)$, and $\mathrm{HBV}$ status $(P=0.048)$ could be clinical adverse prognostic factors for all patients (Table 3); and then multivariable analysis was performed; results showed that IPI $(P=0.002)$, chemotherapy regimens $(P=$ $0.017)$, and $M Y C$ gene arrangements $(P=0.004)$ were independent adverse prognostic factors for all patients in this study.

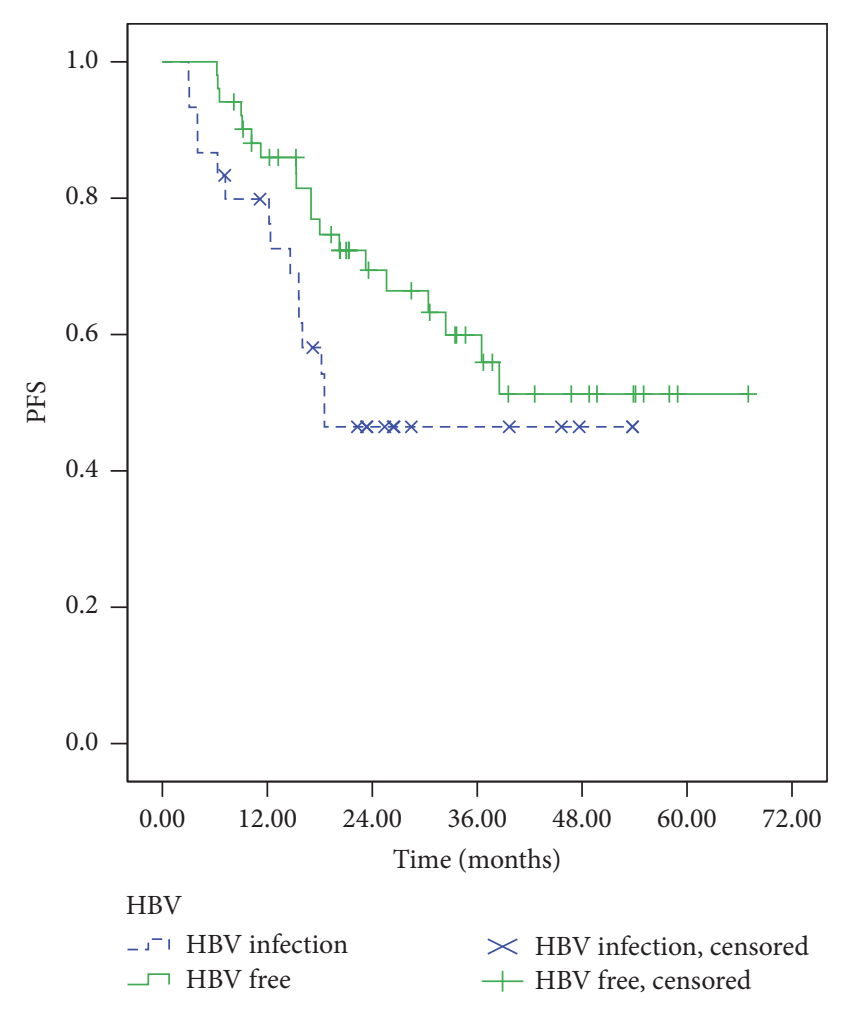

FIGURE 4: The progression-free survival of patients in HBV infection group and $\mathrm{HBV}$-free group.

\section{Discussion}

In this study, we investigated the prognosis and risk factors of DLBCL patients with HBV infection. The results indicated that (1) the incidence of MYC and BCL2 gene rearrangements in $\mathrm{HBV}$ infection group was higher than that in HBV-free group; (2) the median OS and PFS of patients with $\mathrm{HBV}$ infection was worse than those with HBV-free; (3) multivariable analysis indicated that IPI, chemotherapy regimens, and MYC gene rearrangements were independent prognostic factors for DLBCL patients.

The rate of $M Y C$ gene rearrangements was $20 \%$ in patients with $\mathrm{HBV}$ infection, and this result was inconsistent with the previously published articles in which MYC gene rearrangements were detected in almost all cases of BL but in less than $10 \%$ of the DLBCL patients at diagnosis $[13,14]$. For this 


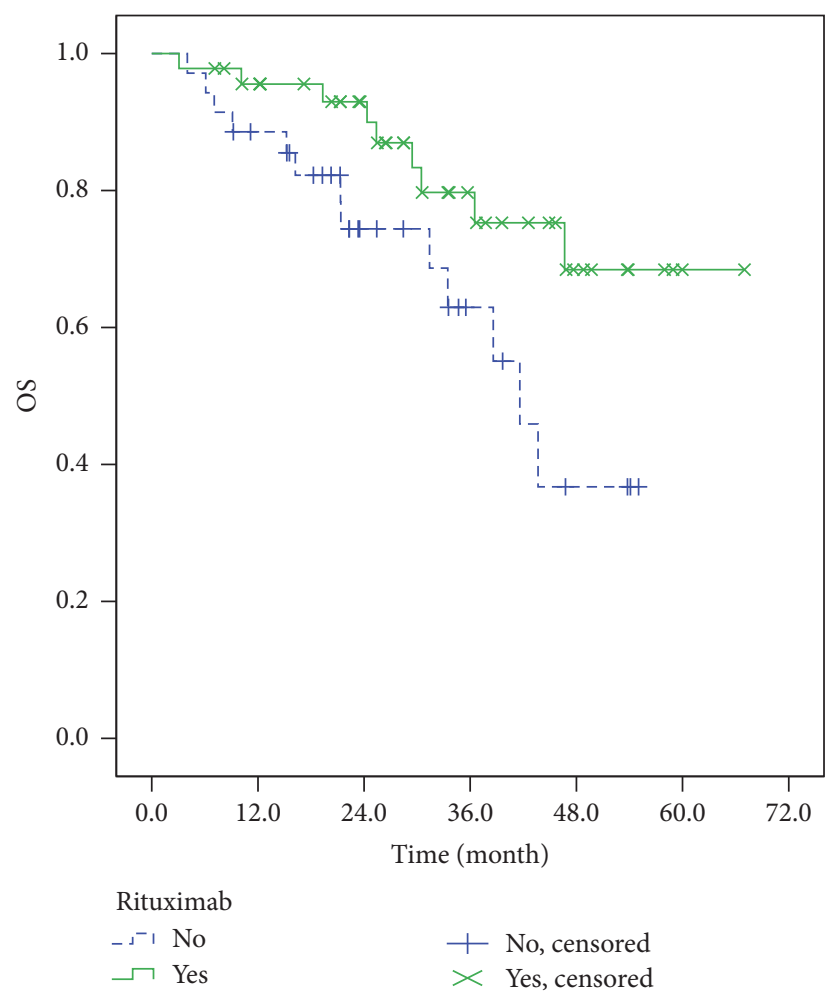

FIGURE 5: The OS patients between rituximab-containing group and rituximab-free group.

difference, we believed that HBV infection may remarkably promote the $M Y C$ gene rearrangements. With regard to the rate of $B C L 2$ gene rearrangements, previous studies reported that the proportion of $B C L 2$ gene rearrangements was from $20 \%$ to $30 \%$ in DLBCL [15]. In this study, the rate of $B C L 2$ gene rearrangements was $23.3 \%$ in patients with $\mathrm{HBV}$ infection in the cohort, which was consistent with the reports mentioned above.

In this cohort, the median OS and PFS of DLBCL patients with HBV infection was shorter than those with HBV-free, and then we further explored potential adverse prognostic factors of DLBCL patients, such as sex, age, clinical stage, IPI, Ki-67, molecular subtype, chemotherapy regimens, $M Y C$ and $B C L 2$ rearrangements, and HBV status; and multivariable analysis showed that IPI, chemotherapy regimens, and $M Y C$ gene rearrangements were independent risk factors for DLBCL patients.

IPI is the most common clinical tool used to evaluate the prognosis of DLBCL patients, which includes five prognostic factors with age, Ann Arbor clinical stage, performance status, serum lactate dehydrogenase, and the number of extranodal sites of disease. Based on these factors, DLBCL patients were stratified into four risk categories, namely, low risk, low intermediate risk, high intermediate risk, and high risk. The four risk groups have significantly different fiveyear OS rates of $73 \%, 51 \%, 43 \%$, and $26 \%$, respectively [16]. A study from China explored the poor prognostic factors of patients with DLBCL, and results showed that IPI 3-5 scores affected OS and PFS of DLBCL patients [17]. In this study, the incidence of patients with IPI $>2$ in HBV infection

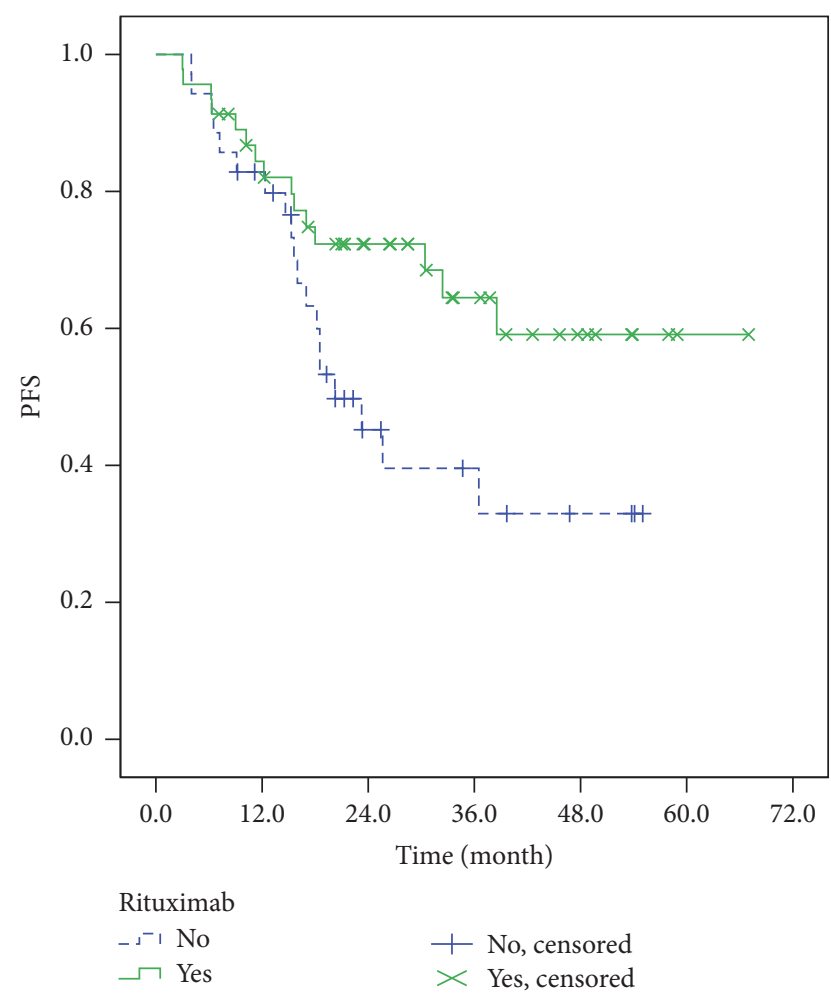

FIGURE 6: The PFS of patients between rituximab-containing group and rituximab-free group.

group was almost identical to that of HBV-free group; in addition, results from multivariable analysis revealed that IPI was an independent adverse prognostic factor for all patients; therefore, we considered that the poor survival of patients in HBV infection group may be associated with IPI.

Rituximab, an anti-CD20 humanized chimeric monoclonal antibody plus CHOP chemotherapy (cyclophosphamide, doxorubicin, vincristine, and prednisolone) is currently a standard treatment of DLBCL. The application of rituximab obviously increases response rates. What is more, there are also improvements in survival. A 10-year retrospective follow-up analysis results indicated that there were significant differences between R-CHOP and CHOP for OS and PFS for all DLBCL patients [18]. Six-year results of an open-label randomized study of the MabThera International Trial (MInT) Group displayed that rituximab-containing chemotherapy improved long-term outcomes for young DLBCL patients with good prognosis [19]. In this study, the rate of patients who received rituximab-containing chemotherapy in HBV infection group was lower than that in HBV-free group; what is more, we further investigated clinical survival of patients between rituximab-containing group and rituximabfree group, and results showed that the median OS and PFS of patients in rituximab-free group was worse than that in rituximab-containing group; thence, we believed that the poor survival of patients with HBV infection was closely involved in chemotherapy regimens.

$M Y C$ gene rearrangements were reported to be involved in inferior survival of DLBCL patients. Kojima et al. explored the association between $M Y C$ rearrangements and the overall 
TABLE 3: Results of the Cox regression to evaluate independent risk factors affecting overall survival of all patients in this study.

\begin{tabular}{lccccc}
\hline Characteristics & HR & $\begin{array}{c}\text { Univariable analysis } \\
95 \% \text { CI of HR }\end{array}$ & $P$ value & HR & $\begin{array}{c}\text { Multivariable analysis } \\
95 \% \text { CI of HR }\end{array}$ \\
\hline Sex & 1.512 & $0.613-3.725$ & 0.369 & & \\
Age $(\geq 60)$ & 0.540 & $0.233-1.251$ & 0.151 & & $0.721-10.638$ \\
Stage (III/IV) & 0.410 & $0.151-1.113$ & 0.080 & \\
IPI (>2) & 3.876 & $1.572-9.524$ & 0.003 & 4.274 & $0.135-0.820$ \\
Ki-67 (>80\%) & 2.036 & $0.602-6.884$ & 0.252 & & $1.706-18.182$ \\
Molecular subtype & 0.605 & $0.222-1.645$ & 0.325 & & 0.017 \\
Rituximab & 0.404 & $0.172-0.949$ & 0.038 & 0.333 & 0.004 \\
MYC positive & 4.274 & $1.553-11.765$ & 0.005 & 5.556 & $0.487-3.034$ \\
BCL2 positive & 0.507 & $0.166-1.545$ & 0.232 & & 0.677 \\
HBV status & 2.338 & $1.006-5.432$ & 0.048 & 1.215 & \\
\hline
\end{tabular}

Note. HR, hazard ratio; CI, confidence index; IPI, international prognostic index.

survival of DLBCL patients, and multivariable analysis showed that $M Y C$ rearrangements were independent adverse prognostic factor [20]. Similarly, Aukema et al. also investigated the influence of $M Y C$ gene rearrangements for the overall survival of DLBCL patients, and the results indicated that patients with $M Y C$ gene rearrangements had a poor survival compared to the patients without the MYC gene rearrangements [21]. Tzankov et al. found that patients with $M Y C$ rearrangements were more likely to be treatmentresistant $(P<0.0001)$ and had a poor prognosis [22]. Our results indicated that the incidence of $M Y C$ gene arrangements was high in DLBCL patients with HBV infection, and it was also an independent adverse prognostic factor for those patients. Therefore, we considered that the poor prognosis of DLBCL patients with HBV infection was closely associated with $M Y C$ gene rearrangements.

The relationship between $B C L 2$ gene rearrangements and the OS of DLBCL patients remains controversial now. Barrans SL reported that DLBCL patients with BCL2 gene rearrangements had a decreased survival compared with those without BCL2 gene rearrangements [23], whereas Kawasaki et al. found that DLBCL patients with BCL2 gene rearrangements had a better survival than those without BCL2 gene rearrangements [24]. In present study, although the rate of $B C L 2$ gene rearrangements was high in DLBCL patients with $\mathrm{HBV}$ infection, multivariable analysis showed that $B C L 2$ gene rearrangements was not an independent adverse prognostic factor. Therefore, we considered that BCL2 gene rearrangements were not involved with the poor prognosis of DLBCL patients with HBV infection.

The incidence of MYC and BCL2 gene rearrangements in $\mathrm{HBV}$ infection group was higher than that in HBV-free group. Some studies found that chronic-phase hepatitis may increase the expression of $c-m y c$ and BCL2 gene $[25,26]$. According to the existing evidences, we considered that $\mathrm{HBV}$ infection may promote $M Y C$ and BCL2 gene rearrangements, but the distinct mechanism is still unclear now.

In conclusion, $M Y C$ and BCL2 gene rearrangements were common in DLBCL patients with HBV infection. Multivariable analysis revealed that IPI, chemotherapy regimens, and $M Y C$ gene rearrangements were independent adverse prognostic factors for DLBCL patients. But, as the limited case number in the study, large-scale multicenter clinical studies are needed to verify the results in our study.

\section{Disclosure}

The funders had no role in study design, data collection, and analysis, decision to publish, or preparation of the manuscript.

\section{Conflicts of Interest}

All authors declare that they have no conflicts of interest.

\section{Acknowledgments}

The study was funded by Science and Technology Department of Jilin Province, China (no. 20140414004GH; no. 20160101010JC) and Jilin Province Development and Reform Commission Department (no. 2014Y090).

\section{References}

[1] h. Zhong, X. Liu, X. Bing, and Z. Za, Chinese Medical Association, the guideline of Prevention and Treatment for Chronic Hepatitis B, vol. 32, Chinese Society of Hepatology and Chinese Society of Infectious Diseases, 2010.

[2] C. W. Shepard, E. P. Simard, L. Finelli, A. E. Fiore, and B. P. Bell, "Hepatitis B virus infection: epidemiology and vaccination," Epidemiologic Reviews, vol. 28, no. 1, pp. 112-125, 2006.

[3] A. Schweitzer, J. Horn, R. T. Mikolajczyk, G. Krause, and J. J. Ott, "Estimations of worldwide prevalence of chronic hepatitis $B$ virus infection: a systematic review of data published between 1965 and 2013," The Lancet, vol. 386, no. 10003, pp. 1546-1555, 2015.

[4] G.-L. Xia, C.-B. Liu, H.-L. Cao et al., "Prevalence of hepatitis B and $\mathrm{C}$ virus infections in the general Chinese population. results from a nationwide cross-sectional seroepidemiologic study of hepatitis A, B, C, D, and E virus infections in China, 1992," International Hepatology Communications, vol. 5, no. 1, pp. 6273, 1996.

[5] S. Dalia, J. Chavez, J. J. Castillo, and L. Sokol, "Hepatitis B infection increases the risk of non-Hodgkin lymphoma: A 
meta-analysis of observational studies," Leukemia Research, vol. 37, no. 9, pp. 1107-1115, 2013.

[6] M. Taborelli, J. Polesel, M. Montella et al., "Hepatitis B and C viruses and risk of non-Hodgkin lymphoma: A case-control study in Italy," Infectious Agents and Cancer, vol. 11, no. 1, article no. 27, 2016.

[7] A. Nath, R. Agarwal, P. Malhotra, and S. Varma, "Prevalence of hepatitis B virus infection in non-Hodgkin lymphoma: a systematic review and meta-analysis," Internal Medicine Journal, vol. 40, no. 9, pp. 633-641, 2010.

[8] L. Deng, Y. Song, K. H. Young et al., "Hepatitis B virus-associated diffuse large B-cell lymphoma: Unique clinical features, poor outcome, and hepatitis B surface antigen-driven origin," Oncotarget, vol. 6, no. 28, pp. 25061-25075, 2015.

[9] W. Guo, W. Zhang, C. Liu, Y. Song, and O. Bai, "Clinical analysis of the HBV infection status of 135 patients with diffuse large B cell lymphoma treated with R-CHOP or CHOP/CHOP-like chemotherapy," PLoS ONE, vol. 10, no. 6, Article ID e0129064, 2015.

[10] D. Norris and J. Stone, WHO classification of tumours of haematopoietic and lymphoid tissues, 2008.

[11] B. D. Cheson, B. Pfistner, M. E. Juweid et al., "Revised response criteria for malignant lymphoma," Journal of Clinical Oncology, vol. 25, no. 5, pp. 579-586, 2007.

[12] R. Reber, Y. Banz, E. Garamvölgyi, A. Perren, and U. Novak, "Determination of the molecular subtypes of diffuse large Bcell lymphomas using immunohistochemistry: a case series from the Inselspital, Bern, and a critical appraisal of this determination in Switzerland.," Swiss Medical Weekly, vol. 143, p. w13748, 2013.

[13] H. A. Poirel, M. S. Cairo, N. A. Heerema et al., "Specific cytogenetic abnormalities are associated with a significantly inferior outcome in children and adolescents with mature B-cell nonHodgkin's lymphoma: results of the FAB/LMB 96 international study," Leukemia, vol. 23, no. 2, pp. 323-331, 2009.

[14] K. J. Savage, N. A. Johnson, S. Ben-Neriah et al., "MYC gene rearrangements are associated with a poor prognosis in diffuse large B-cell lymphoma patients treated with R-CHOP chemotherapy," Blood, vol. 114, no. 17, pp. 3533-3537, 2009.

[15] M. G. Tibiletti, V. Martin, B. Bernasconi et al., "BCL2, BCL6, MYC, MALT 1, and BCL10 rearrangements in nodal diffuse large B-cell lymphomas: a multicenter evaluation of a new set of fluorescent in situ hybridization probes and correlation with clinical outcome," Human Pathology, vol. 40, no. 5, pp. 645-652, 2009.

[16] M. Shipp, D. Harrington, and J. Anderson, "A predictive model for aggressive non-Hodgkin's lymphoma. the international Non-Hodgkin's Lymphoma Prognostic Factors Project," The New England Journal of Medicine, vol. 329, no. 14, pp. 987-994, 1993.

[17] J. Wen, J. Zhou, Z. Liu, T. Liu, and C. Xu, "Analysis of survival and prognosis in 409 newly diagnosed patients with diffuse large B-cell lymphoma," Chinese Journal of Hematology, vol. 35, no. 4, pp. 318-324, 2014.

[18] X. Li, Z. Liu, J. Cao et al., "Rituximab in combination with CHOP chemotherapy for the treatment of diffuse large B cell lymphoma in China: A 10-year retrospective follow-up analysis of 437 cases from Shanghai Lymphoma Research Group," Annals of Hematology, vol. 91, no. 6, pp. 837-845, 2012.

[19] M. Pfreundschuh, E. Kuhnt, L. Trümper et al., "CHOP-like chemotherapy with or without rituximab in young patients with good-prognosis diffuse large-B-cell lymphoma: 6-year results of an open-label randomised study of the MabThera International Trial (MInT) Group," The Lancet Oncology, vol. 12, no. 11, pp. 1013-1022, 2011.

[20] M. Kojima, H. Nishikii, J. Takizawa et al., "MYC rearrangements are useful for predicting outcomes following rituximab and chemotherapy: Multicenter analysis of Japanese patients with diffuse large B-cell lymphoma," Leukemia \& Lymphoma, vol. 54, no. 10, pp. 2149-2154, 2013.

[21] S. M. Aukema, M. Kreuz, C. W. Kohler et al., "Biological characterization of adult MYC-translocation-positive mature B-cell lymphomas other than molecular Burkitt lymphoma," Haematologica, vol. 99, no. 4, pp. 726-735, 2014.

[22] A. Tzankov, Z. Y. Xu-Monette, M. Gerhard et al., "Rearrangements of MYC gene facilitate risk stratification in diffuse large B-cell lymphoma patients treated with rituximab-CHOP," Modern Pathology, 2013.

[23] S. L. Barrans, P. A. S. Evans, S. J. M. O’Connor et al., "The $t(14 ; 18)$ is associated with germinal center-derived diffuse large B-cell lymphoma and is a strong predictor of outcome," Clinical Cancer Research, vol. 9, no. 6, pp. 2133-2139, 2003.

[24] C. Kawasaki, K. Ohshima, J. Suzumiya et al., "Rearrangements of bcl-1, bcl-2, bcl-6, and c-myc in diffuse large B-cell lymphomas," Leukemia \& Lymphoma, vol. 42, no. 5, pp. 1099-1106, 2001.

[25] Y. Fujimoto, Y. Ishizaka, T. Tahira et al., "Possible involvement of c-myc but not ras genes in hepatocellular carcinomas developing after spontaneous hepatitis in LEC rats," Molecular Carcinogenesis, vol. 4, no. 4, pp. 269-274, 1991.

[26] V. E. Karev, "Fas, FasL, and bcl-2 expression on hepatic intralobar lymphocytes in different variants of the natural course of chronic HBV and HCV infection and in its outcomes," Arkhiv Patologii, vol. 76, no. 1, pp. 16-21, 2014. 


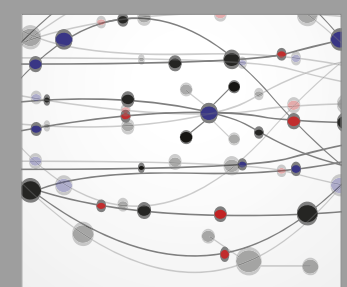

The Scientific World Journal
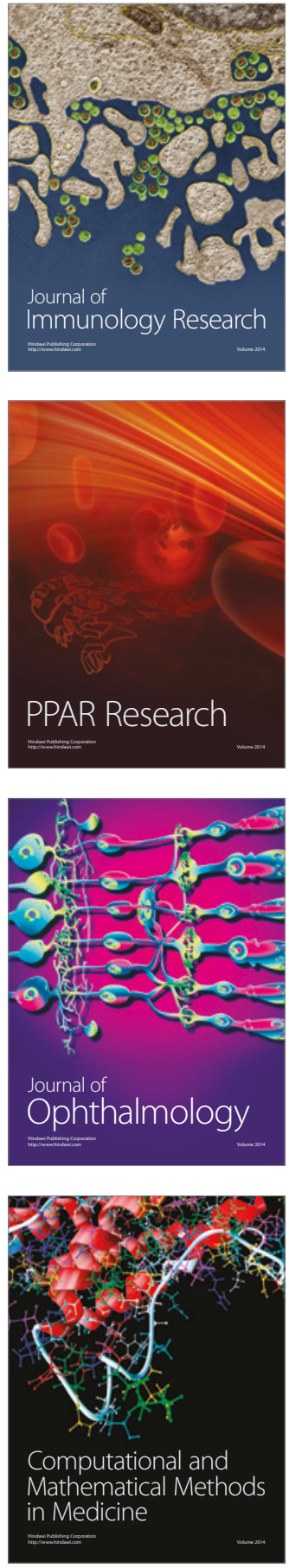

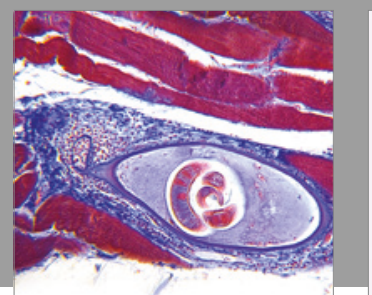

Gastroenterology Research and Practice
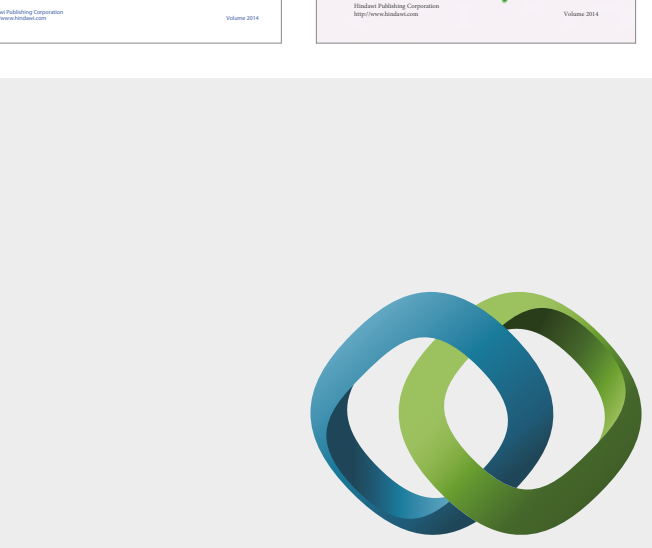

\section{Hindawi}

Submit your manuscripts at

https://www.hindawi.com
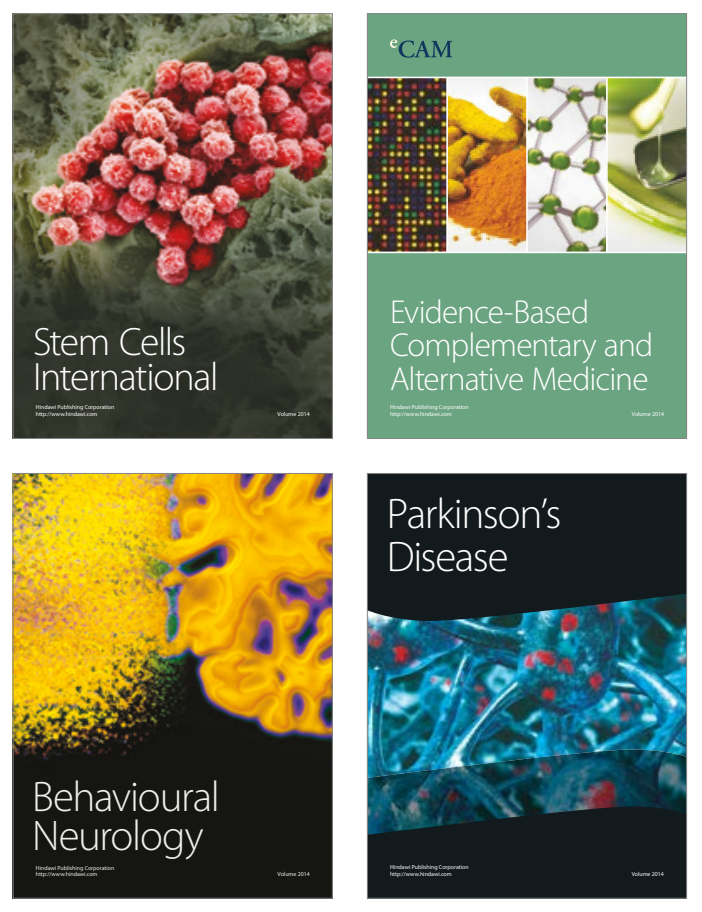
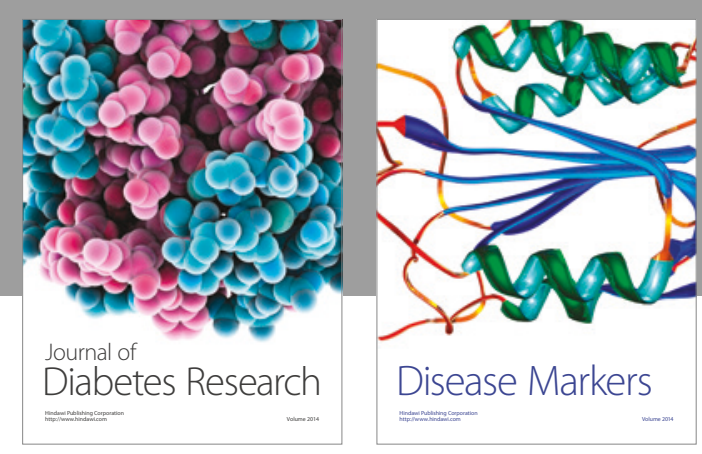

Disease Markers
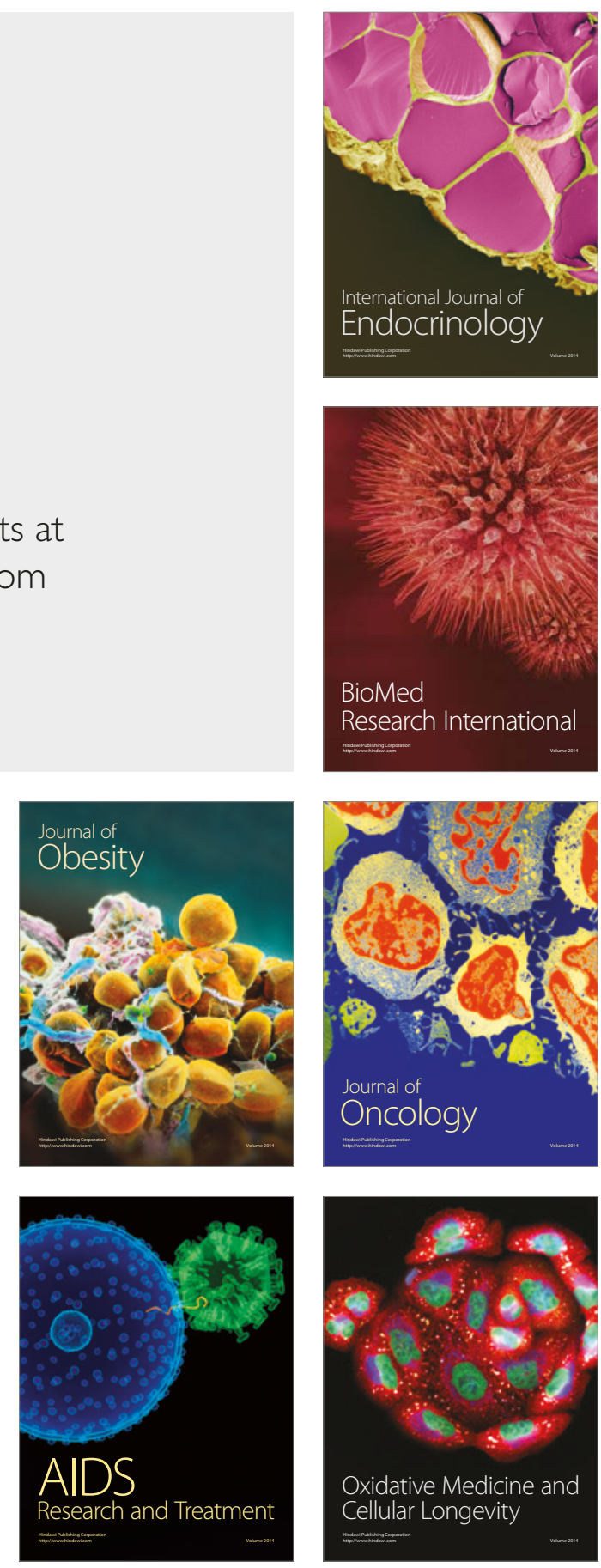